\title{
DEPRESSION, ADOLESZENZ UND AGENCY - ZUR (RE-) KONSTRUKTION DEPRESSIVER IDENTITÄTEN AN DER SCHNITTSTELLE VON PRIMÄRER LEBENSPRAXIS UND STELLVERTRETENDER KRISENBEWÄLTIGUNG
}

Christian Armbrüster

Johannes Gutenberg-Universität Mainz, Institut für Erziehungswissenschaft

E-Mail: armbruester@uni-mainz.de

URL: https://www.sozialpaedagogik.fb02.uni-mainz.de/team/christian-armbruester-mal

Zitationsvorschlag:

Armbrüster, Christian (2021): Depression, Adoleszenz und Agency - Zur (Re-)Konstruktion depressiver Identitäten an der Schnittstelle von primärer Lebenspraxis und stellvertretender Krisenbewältigung. In: Gesellschaft - Individuum - Sozialisation (GISo). Zeitschrift für Sozialisationsforschung, 2 (1). DOl: 10.26043/GISo.2021.1.4

Link zum Artikel:

https://doi.org/10.26043/G/So.2021.1.4 


\title{
DEPRESSION, ADOLESZENZ UND AGENCY - ZUR (RE-) KONSTRUKTION DEPRESSIVER IDENTITÄTEN AN DER SCHNITTSTELLE VON PRIMÄRER LEBENSPRAXIS UND STELLVERTRETENDER KRISENBEWÄLTIGUNG
}

\author{
Christian Armbrüster
}

Abstract: Die vergleichende Einzelfallstudie analysiert institutionell eingeforderte biographische Berichte Adoleszenter mit der Diagnose einer Depression. Sie untersucht, wie sich diese als Narrativ im Kontext von Identitätsbildung und lebenspraktischem Wirklichkeitsvollzug darstellt. In den Eigenberichten der Jugendlichen und jungen Erwachsenen lässt sich Depression als kohärenzstiftende und vermittelnde (Teil-)Identität rekonstruieren. Diese steht in enger Beziehung zu akkumulierten traumatischen Krisen der (familiären) Lebenspraxis, sich daraus ableitenden Handlungsroutinen sowie relational erzeugten Deutungsmustern an der Schnittstelle zur psychosozialen Hilfeinstanz. In diesem Wechselverhältnis von lebenspraktischer Krisenbewältigung und relationaler Identitätsarbeit wird Agency in ihrer inneren und äußeren Struktur sichtbar. Dabei präsentiert der Beitrag die triangulative Verknüpfung der Perspektiven von Identität, Lebenspraxis und Agency als aussichtsreichen Zugang für die fallrekonstruktive Forschung.

Keywords: Identität, Lebenspraxis, Agency, Fallrekonstruktion, Depression, Adoleszenz

\section{EINLEITUNG}

Weltweit ist Depression die vierthäufigste Ursache für Krankheit und Behinderung bei Jugendlichen im Alter von 15 bis 19 Jahren (WHO 2019). Im europäischen Vergleich ist die Prävalenz depressiver Symptomatik bei Jugendlichen und jungen Erwachsenen (15 bis 29 Jahre) in Deutschland mit am höchsten (11,5\%); deutlich höher als der EU-Durchschnitt von 5,2 \% (Hapke et al. 2019, 64). Zugleich bilden die sog. affektiven Störungen die am schnellsten zunehmende F-Diagnosegruppe (Dilling et al. 2015) im Jugendalter, ebenfalls angeführt von den depressiven Störungen (Steffen et al. 2019, 21). ${ }^{1}$ Damit stellt sich Depression als eine der bedeutsamsten und bedrohlichsten Erkrankungen im Jugendund jungen Erwachsenenalter dar. Da sie in der "Gefahrenzone" (Döbert et al. 1980, 9) der Adoleszenz inmitten des Sozialisationsprozesses auftritt, erscheint die Analyse von deren komplexen Verflechtungen als besondere Herausforderung für die Sozialisationsforschung. Diese ist einerseits an der interaktiven Herstellung, Aufrechterhaltung und Transformation ,von kognitiven, motivationalen und affektiven Kompetenzen, die auf die performative Lebensführung Einfluss nehmen“ (Bauer 2016, 80) interessiert, während sie ebenso die diese Prozesse umspannenden sozialisatorischen Krisenverläufe und Instanzen (Oevermann 2016) sowie übergrei-

\footnotetext{
1 Die aktuell gültige Fassung der Internationalen statistischen Klassifikation der Krankheiten und verwandter Gesundheitsprobleme (ICD-10; Dilling et al. 2015) führt psychische und Verhaltensstörungen in Kapitel V auf. Der Buchstabe F bedeutet, dass ein Hinweis auf eine psychische Störung vorliegt. Die affektiven Störungen bilden hierin eine Gruppe (F30-F39), deren gemeinsames verbindendes Merkmal insbesondere eine klinisch bedeutsame Veränderung der Stimmungslage oder Affektivität ist (ebd., 159). Die (unipolaren) depressiven Störungen werden dieser Gruppe in der psychiatrischen Klassifikation zugeordnet und fallen unter den Schlüssel F32 bzw. im Falle sich wiederholender Depressionen unter F33. Die ICD-10 benennt drei typische Symptome der Depression: depressive Stimmung, Verlust von Interesse und Freude sowie eine erhöhte Ermüdbarkeit. Daneben werden sieben andere häufige Symptome wie verminderte Konzentration und Aufmerksamkeit, vermindertes Selbstwertgefühl und Selbstvertrauen, Schuldgefühle und Gefühle von Wertlosigkeit, negative und pessimistische Zukunftsperspektiven, Suizidgedanken, erfolgte Selbstverletzung oder Suizidhandlungen, Schlafstörungen und verminderter Appetit aufgeführt.
} 
fenden Schematisierungen sozialer Erfahrungen im Sinne von Identitätsbildungsprozessen (Keupp et al. 2008; Leipold/Greve 2015) in den Blick nimmt. Die Historie der Sozialisationsforschung kennzeichnet dabei ein Paradigmenwechsel vom „akteurlosen Strukturalismus der 1960er und 70er Jahre“ über „einen strukturlosen Subjektzentrismus der 80er und 90er Jahre" (Bauer 2016, 82) hin zu neueren Ansätzen, die eine Überwindung dualistischer Betrachtungsweisen von Struktur und (individueller) Agency anstreben und vielmehr Beziehungsgeflechte fokussieren, in denen sich diese, in wechselseitigen Prozessen, überhaupt erst als solche konstituieren (z. B. Hungerland/Kelle 2014; Esser et al. 2016; Grundmann 2017). Jenem Zugang, der mit dem Begriff relational verbunden ist, folgend, nähert sich der vorliegende Beitrag Depression im Jugend- und jungen Erwachsenenalter, indem er versucht, zu einem besseren Verständnis des Phänomens - im Wechselverhältnis von Lebenspraxis, Identität und Agency - beizutragen. Qualitativ-rekonstruktive Studien, die sich mit Dimensionen von Biographie und Lebenspraxis (Riemann 1987; Steffens 2004), Identität und Agency (Hoffmann 2011, 2012, 2015; Deppermann 2015) im Kontext von psychischer Erkrankung befassen, liegen bislang nur vereinzelt vor. Eine systematische Zusammenführung dieser Dimensionen findet dabei ebenso wenig Berücksichtigung wie biographische Erzählungen von Adoleszenten. An diesen Desideraten setzt die Fallanalyse an. Ausgangspunkt bilden zwei Eigenberichte von Adoleszenten mit der Diagnose einer depressiven Störung, welche diese im Vorfeld einer stationären psychosomatischen Behandlung verfasst haben. Für die Analyse sind insbesondere folgende Forschungsfragen handlungsleitend:

1. Wie kann Depression in biographischen Identitätskonstruktionen Adoleszenter, im Rahmen institutionell eingeforderter Eigenberichte, verstanden werden?

2. Wie zeigt sich Depression in den sozialisatorischen Krisenbewältigungsprozessen der Lebenspraxen, die sich in diesen Protokollen abbilden?
3. Wie stellt sich in diesem Kontext Agency - als Handlungsfähigkeit und Wirkmächtigkeit dar?

Der Beitrag gliedert sich dabei wie folgt: Auf die Ausführung der theoretischen Perspektiven von Lebenspraxis, Identität und Agency (2.) folgt die Darstellung des fallrekonstruktiven Zugangs zum Material (3.). Im Zentrum steht die vergleichende Analyse der biographischen Erzählungen von „Paula" und „Elena“ (4.). Abschließend findet sich ein Resümee der wichtigsten Ergebnisse ebenso wie ein Ausblick auf weiterführende Forschungsperspektiven.

\section{AGENCY, LEBENSPRAXIS UND IDENTITÄT ALS THEORETISCHE ZUGÄNGE}

Mit dem Begriff der Lebenspraxis beschreibt UIrich Oevermann zunächst jene „Einheit, der als lebendiger, als Agens" die "Strukturtransformationsprozesse der Erzeugung des Neuen zuzurechnen sind" (2016, 157). Wenn hier von Strukturtransformation die Rede ist, so ist damit insbesondere der Prozess humaner Sozialisation angesprochen, den Oevermann als Wechselspiel von Krise und Routine begreift und innerhalb dessen sich die Lebenspraxis - $d$. h. ein einzelner Mensch oder eine Gruppe von Menschen - als gestalterisches Element derart einbringt, dass sie Krisen entweder durch Rückgriff auf bewährtes, routinisiertes Wissens oder, falls dies nicht ausreicht, durch die Erzeugung von Neuem bewältigt (Garz/Raven 2015, 26 ff.). Die Krisenhaftigkeit des Sozialisationsprozesses leitet sich, nach Oevermann, bereits aus dem Umstand $a b$, dass Menschen über strukturierte Handlungsspielräume verfügen, die sie gleichzeitig dem Zwang aussetzen, in einer "Zukunftsoffenheit von Entscheidungsalternanten“ (Oevermann 2016, 159) auswählen und diese Entscheidungen zudem noch begründen zu müssen (ebd.). Den Gedanken folgend, dass Lebenspraxis in ihrem Vollzug von Wirklichkeit Autonomie und Handlungsfähigkeit sowohl konstituiert (Oevermann 2000, 130 f.) als auch für weitere Krisenbewältigungen immer wieder erfordert, wie auch der Umstand, dass sie im Krisenbewältigungsprozess auf übergreifende Interaktions- und Handlungsbezüge rekurriert (Oevermann 2016, 160) und diese durch ihr Handeln formt, führt sogleich zu den weiteren 
beiden Konzepten von Identität und Agency. Zur Bestimmung des Identitätsbegriffs wird auf das Konzept der Identitätskonstruktionen von Heiner Keupp et al. (2008) Bezug genommen, welches gesellschaftliche Wandlungsprozesse der Spätmoderne - die mit Begriffen wie Individualisierung, Pluralisierung, Globalisierung assoziiert sind und in die Adoleszenten in besonderer Weise eingebunden sind - zentral berücksichtigt. Identität wird hier in erster Linie als konzeptioneller Rahmen verstanden, „innerhalb dessen eine Person ihre Erfahrungen interpretiert" (Keupp 2005, 809) und die jeweils die Basis für die alltägliche Identitätsarbeit bildet (ebd.). Der Begriff der Identitätsarbeit verweist auf die Tatsache, dass hier eine (soziale) Konstruktionsleistung adressiert wird, welche auf die Produktion einer "individuellen sozialen Verortung" (Keupp 2000) zielt, d. h. der Herstellung einer Passung zwischen dem subjektiven "Innen“ und dem gesellschaftlichen „Außen“ (ebd.). Die Passung wird dabei u. a. über die Verknüpfung unterschiedlicher Teilidentitäten innerhalb eines machtbestimmten sozialen Raums und unter Rückgriff auf individuell-biographisch begründete Kompetenzen - über die kommunikativ vermittelten Netzwerkressourcen bis hin zu gesellschaftlich-institutionell vermittelten Ideologien und Strukturvorgaben - hergestellt und begründet sich zentral in der Narration, also im sozialen Aushandlungs- und Darstellungsprozess mit Anderen (Keupp et al. 2008, 189 ff.). Durch die Syntheseleistungen der Kohärenz, des Erlangens von Anerkennung - bei gleichzeitigem Erhalt von Autonomie - ebenso wie dem Erleben von Authentizität, verdichten sich dominierende Teilidentitäten, biographische Kernnarrationen und ein Identitätsgefühl zu dem, was Keupp et al. als Meta-Identität bezeichnen (ebd., 218). Da die
Sicherung von Handlungsfähigkeit durch Identitätsarbeit auch in ihrer Konzeption eine zentrale Rolle spielt (ebd., $235 \mathrm{ff}$.), soll, als drittes, das dieser Arbeit zugrunde liegende Konzept von Agency näher erläutert werden. Hier erweist sich die Konzeptionierung von Mustafa Emirbayer und Jeff Goodwin und von Emirbayer und Ann Mische als besonders anschlussfähig. Menschliche Agency zeichnet ihnen zufolge insbesondere die Fähigkeit sozial eingebetteter Akteur*innen aus, „sich vorgefundene kulturelle Kategorien und Bedingungen des Handelns in Einklang mit ihren persönlichen und kollektiven Idealen, Interessen und Verpflichtungen anzueignen, sie zu reproduzieren und potentiell Innovationen einzuführen“" (Emirbayer/Goodwin 2017, 324). Bei der Konstitution von Handlungsfähigkeit unterscheiden die Autoren drei Dimensionen: (1.) Iteration: Eine habituelle Dimension, die sich auf vergangene Erfahrungen bezieht, (2.) Projektion: eine an der Zukunft orientierte Fähigkeit, alternative Möglichkeiten zu imaginieren sowie (3.) praktische Evaluation: die Fähigkeit, mögliche Handlungsverläufe in der Gegenwart praktisch und normativ zu prüfen und zu beurteilen (Emirbayer/Mische 2017). Dabei fokussieren Emirbayer und Mische wie auch Emirbayer und Goodwin, wie zu sehen, stark auf eine innere Strukturiertheit von Agency. Da alle drei der hier vorgestellten Zugänge von einer temporal-relationalen Herstellung der von ihnen behandelten Konzepte ausgehen, ergeben sich mehrere theoretische Anknüpfungspunkte, die im vorliegenden als Bezugsrahmen für die empirische Analyse nutzbar gemacht werden sollen und deren Verbindung vielversprechend erscheint, weil Lebenspraxis, Identität und Agency als in einem gegenseitigen Wechselverhältnis eingebunden betrachtet werden können: 
Abbildung 1: Wechselseitige Herstellung und Anrufung von Identität, Lebenspraxis und Agency

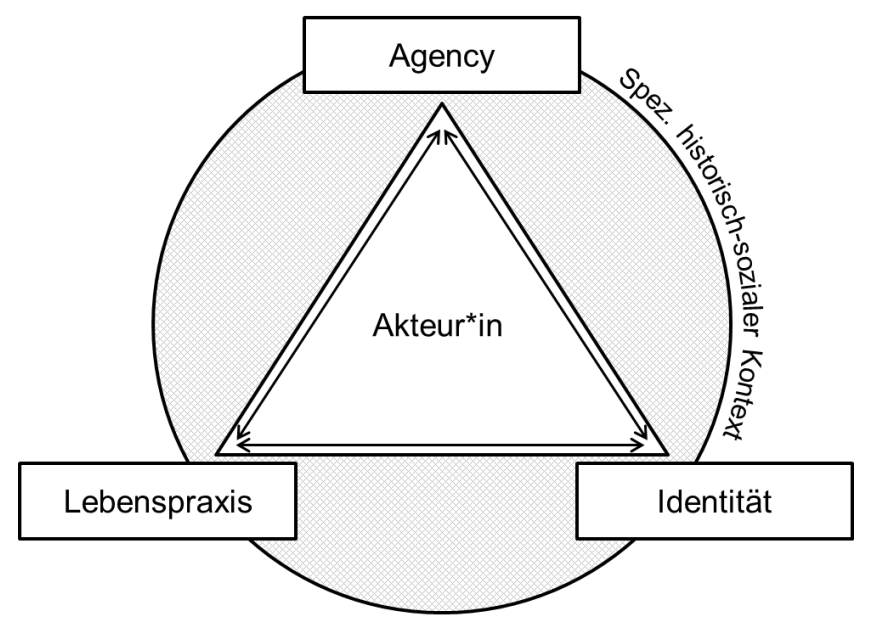

Quelle: eigene Darstellung

\section{METHODE UND DATENBASIS}

Das Datenmaterial der Fallrekonstruktion bilden biographische Erzählungen von Jugendlichen und jungen Erwachsenen, welche diese in einem sog. Eigenbericht im Vorfeld einer stationären psychosomatischen Behandlung schriftlich verfasst haben. Berücksichtigt werden muss, dass die Datensammlung nicht originär zu Forschungszwecken stattfand (Oevermann 2000, 87 f.). Es handelt sich bei den Protokollen um einen Bestandteil der routinisierten (Be-)Handlungspraxis der Jugendpsychosomatik und soll dieser - nach eigenen Angaben - einen Überblick über die Person und deren Krankheitsbild ebenso wie eine bessere Behandlungsplanung ermöglichen. Die Patient*innen sollen das Verfassen des Dokuments zu einer Reflexion und Zielformulierung hinsichtlich der therapeutischen Behandlung anhalten. ${ }^{2}$ Der Eigenbericht folgt einem (teil-)standardisierten Format, das von der Klinik vorgegeben ist, wird von allen zukünftigen Patient*innen der Klinik bearbeitet und umfasst vier Themenfelder, deren Beantwortung je eine Seite nicht überschreiten soll. Diese lauten:

1. Kurze Schilderung Ihrer Lebensgeschichte.
2. Schilderung der zugrunde liegenden Problematik einschließlich der Benennung wichtiger psychischer (a.) und körperlicher (b.) Vorerkrankungen einschließlich der einzunehmenden Medikamente (c.) und der Benennung eventuell bestehender Abhängigkeiten (d.) oder der Einnahme von Suchtmitteln (e.).

3. Kraftquellen, Ressourcen: Was ist gut an mir? Was ist bewahrenswert? Was soll sich während des stationären Aufenthaltes nicht ändern?

4. Was soll in einer stationären Therapie in der [Klinik $A]$ in der für Sie zur Verfügung stehenden Zeit herauskommen? Was erwarten Sie von uns? Was soll nicht passieren? (Leitfaden Eigenbericht)

Insgesamt wurden sieben Eigenberichte für das Forschungsvorhaben gesammelt. Alle Adoleszenten wurden mündlich und schriftlich über die wissenschaftliche Verwendung ihrer Eigenberichte informiert und haben ihr Einverständnis erklärt. Sie wurden darüber aufgeklärt, dass die wissenschaftliche Forschung nicht mit der psychotherapeutischen Behandlung in der Klinik verbunden ist. Auch forschungspraktisch wurde dieser (potentiellen) Machtasymmetrie dadurch begegnet, dass ausschließlich Eigenberichte von

\footnotetext{
2 Die Institution der stationären Jugendpsychosomatik und -psychotherapie in Rehabilitationskliniken lässt sich den psychosozialen Hilfen des komplementären Sektors des Medizin- bzw. Krankenversorgungssystems zuordnen (Schleiffer 2010) und richtet sich an Jugendliche und junge Erwachsene zwischen 16 und 21 Jahren. Ihr Hilfsangebot hat die Wiederherstellung von psychischer und körperlicher Gesundheit sowie gesellschaftlicher Teilhabe, insbesondere im Arbeits- und Ausbildungssektor, zum Ziel.
} 
Patient*innen verwendet wurden, die zum Zeitpunkt der Datenerhebung am Ende ihrer Behandlung in der psychosomatischen Klinik standen. Die vorliegende Auswahl für die vergleichende Fallanalyse (Oevermann 2000, 99 f.) der Berichte von zwei Patientinnen leitete sich aus dem Fokus der Untersuchungsfragen ab. Zentrale Kriterien, neben dem Lebensalter, waren die Behandlungsdiagnose der Depression sowie ein ausreichend hoher Verdichtungsgrad der Protokolle (ebd., 77 ff.), welcher eine Analyse von Identitäts- und Agencykonstruktionen ebenso wie lebenspraktischer Wirklichkeitsvollzüge ermöglichen sollte. Die Analyse fokussiert dabei das erste Themenfeld der (kurzen) Schilderung der Lebensgeschichte. Zur Auswertung der biographischen Erzählungen wird im Folgenden eine Verknüpfung der forschungsmethodischen Zugänge von objektiver Hermeneutik (Oevermann 2000, 2002), der Rekonstruktion narrativer Identität nach Gabriele Lucius-Hoene und Arnulf Deppermann (2002) sowie der AgencyAnalyse (Lucius-Hoene 2012) angestrengt. Dieses Vorgehen begründet sich aus den theoretischen Vorüberlegungen. So betrachtet Oevermann, ausgehend von der Prämisse, dass die soziale Wirklichkeit einer Lebenspraxis nur als Sequenzfolge - d. h. „als ein regelgeleiteter Ablauf und ein Ineinanderübergehen von Vergangenem, Gegenwärtigem und Zukünftigem" (Garz/Raven 2015, 139) - zu verstehen ist, die Methode der sequenzanalytischen Rekonstruktion als diejenige, die dieser Wirklichkeit entspricht (Oevermann 2009, 44). Anhand der detaillierten Analyse der konkreten Beschließungen vorgängig eröffneter Möglichkeiten durch die Lebenspraxis rekonstruiert das Verfahren sukzessive die daraus erschließbare objektive Sinnstruktur eines Falles (Oevermann 2002, 7). Demgegenüber geht es dem Verfahren zur Rekonstruktion narrativer Identität um eine adäquate Erfassung jener subjektiven Bedeutungsstrukturen von Selbstverständigung und -entwurf, die (der Lebenspraxis) Handlungsund Orientierungsfähigkeit sichern (LuciusHoene/Deppermann 2002, 51). Als zentralen Modus dieser Selbstverständigung und der Interpretation der eigenen Erfahrungen begreifen die Autor*innen die autobiographische Narration, während sie unter narrativer Identität diejenigen Aspekte von Identität verstehen, die in diesem Modus dargestellt und hergestellt werden (ebd.,
47). Die „Art und Weise, wie ein Mensch in konkreten Interaktionen Identitätsarbeit als narrative Darstellung und Herstellung von jeweils situativ relevanten Aspekten seiner Identität leistet" (ebd., 55), kann, ihren Ausführungen zufolge, in der systematisch-interpretativen Analyse aus Protokollen autobiographischer Erzählungen anhand der Parameter der temporalen, sozialen und selbstbezüglichen Dimension der narrativen Identität rekonstruiert werden (ebd., 56 ff.). Agency betrachten die Autor*innen dabei als einen wesentlichen Aspekt der (temporalen) historisch-biographischen Konstitution von narrativer Identität, der sich daraus ergibt, wie die erzählende Person ihre Handlungsmöglichkeiten und Handlungsinitiative im Hinblick auf die Ereignisse ihres Lebens linguistisch konstruiert (ebd., 59). Das subjektive Erleben von Handlungsfähigkeit und Wirkmächtigkeit lässt sich, Lucius-Hoene (2012) zufolge, mittels mikrosprachlicher Heuristiken auf drei Analyseebenen differenziert rekonstruieren: 1.) auf der Ebene der Erzählsätze durch die Wahl von Prädikaten, semantischen Rollen und anderen Strategien der Handlungscharakterisierungen, 2.) auf der Ebene der Interaktion mit der Zuhörerschaft hinsichtlich der Gestaltung der kommunikativen Rollen und des Anspruchs auf epistemische Autorität und 3.) auf der Ebene der Leistung des Erzählten für die Bewältigung und Identitätsarbeit der erzählenden Person (ebd., 43). Gleichwohl lässt sich Agency in ihrer äußeren Strukturiertheit - anhand der Analyse von Eröffnungs- und Beschließungsprozeduren (Oevermann 2002, 7) - als Bestandteil des Vollzugs von Wirklichkeit durch die Lebenspraxis rekonstruieren. Im Folgenden wird die Auswertung der Daten mithilfe des hier vorgestellten Zugangs präsentiert. Alle Daten werden in anonymisierter Form vorgestellt.

\section{FALLANALYSE}

\subsection{Der Fall Paula}

Zum Zeitpunkt der Verschriftlichung des Eigenberichts ist Paula 17 Jahre alt. Sie lebt gemeinsam mit ihrem Vater in dessen Wohnung im ländlichen Raum. Paula hat zwei Halbbrüder. Sie hat einen mittleren Schulabschluss und besucht eine Fachoberschule. Sie hat bereits einmal eine psychotherapeutische Behandlung in der Ambu- 
lanz einer Kinder- und Jugendpsychiatrie in Anspruch genommen und ist zum Erhebungszeitpunkt aufgrund einer mittelgradigen depressiven Episode (F32.1) in der Jugendpsychosomatik in Behandlung. ${ }^{3}$

Ihre biographische Erzählung beginnt sie wie folgt:

„Als Kind habe ich recht einfach gelebt in einem kleinen Haus in einem kleinen Dorf namens [ADorf], da hatte ich relativ viele Freunde und viel Spaß, trotz, dass ich einige Zeit in der Grundschule von anderen Schülern ausgeschlossen wurde."

Paula stellt sich in der Eingangssequenz agentiv dar. In der Schilderung ihrer Lebenssituation im Kindesalter tritt sie in der handlungsvollziehenden Rolle auf. Sie beschreibt einen „einfachen“ Lebensstandard, in einem kleinen Dorf, mit vielen Freund*innen sowie ein Lebensgefühl, das sich durch „viel Spaß“ auszeichnet und welches auch Widerstände - wie ein Ausschluss durch andere im Schulkontext - nicht gravierend beeinträchtigen. Mit Blick auf ihre frühe Geschichte zeichnet sie in der Einleitung das Bild einer "heilen Welt". Dann schreibt sie weiter:

„Zu der Zeit als ich in der dritten Klasse war, trennten sich meine Eltern. Das war für mich nie wirklich ein Problem, da sie sich eh immer viel gestritten hatten. Ab da fing alles an, Berg ab zu gehen."

Paula berichtet von der Trennung ihrer Eltern während sie die dritte Klasse der Grundschule besucht und negiert sogleich, dass diese Trennung jemals problematisch für sie gewesen sei. Damit reagiert sie vorwegnehmend auf potentiell normative Vorstellungen der Leser*innen, wonach eine elterliche Trennung für das Kind problemhaft sein könnte, und weist sich sowohl auf der interaktiven Ebene als vorausschauende Erzählerin wie auch auf der Erzählebene als resiliente Akteurin aus. Ihre Ausführung begründet sie mit der Feststellung, dass die Eltern sich „eh immer viel gestritten“ haben, was als Ausdruck von
Verständnis und Akzeptanz für eine vernünftige Entscheidung zum Beenden der konflikthaften Paarbeziehung erscheint. Zugleich wird jedoch im Folgesatz deutlich, dass das „heile Welt“-Narrativ, das die Einleitung der Erzählung bildet, mit der elterlichen Trennung bricht, welche den Beginn einer negativen Verlaufskurve (Schütze 1982, 580) zu markieren scheint.

Anschließend berichtet Paula vom Auszug des Vaters aus der gemeinsamen Wohnung und dessen Wunsch, zu einem späteren Zeitpunkt wieder in den Haushalt einzuziehen. Ihre Mutter habe eine neue Beziehung begonnen und vorgehabt - mit Paula zusammen - zu ihrem neuen Partner zu ziehen. Dabei unterstellt Paula der Mutter die Absicht, sie zu diesem Zeitpunkt aus ihrem „Umfeld rausziehen“ zu wollen. Zudem habe Paule „kein gutes Gefühl“ bei dem neuen Lebenspartner gehabt, da er und sein Sohn sie „nie gut behandelt“ hätten. An Weihnachten im Jahr 2009 sei sie daher zu ihrem Vater gezogen. Von diesem habe sich gut behandelt gefühlt, doch sei er aufgrund von Schichtarbeit häufig abwesend gewesen, weshalb sich die Nachbarsfamilie intensiv um Paula gekümmert habe. Ihr Vater habe häufig wechselnde Partnerinnen gehabt, „da er nicht alleine sein wollte“.

„Dann fand er Maria, anfangs war alles super. [... Doch nach und nach merken wir, dass irgendwas nicht stimmte, sie hetzte immer wieder Papa gegen mich auf [...] und schottete uns total ab. Sie war immer schlimmer werdende Alkoholikerin, das merkten wir leider zu spät. Obwohl Papas Eltern und viele andere Papa gewarnt hatten."

Paulas Erzählung fährt mit dem erfolgreichen „Finden“ einer Lebenspartnerin durch den Vater fort. Die Einleitung „anfangs war alles super“ weist dabei bereits auf einen Wandel im weiteren Verlauf des Geschehens hin, von dem sie sogleich berichtet. In der Erzählung wechselt Paula in die Wir-Perspektive, die sie und ihren Vater als handelnde Akteur*innen umschließt. Die VaterTochter-Dyade beginnt zu merken, dass mit der Lebenspartnerin des Vaters „irgendwas nicht

\footnotetext{
${ }^{3}$ Die ICD-10 unterscheidet die depressiven Episoden nach Schweregrad; von leicht (F32.0), über mittelgradig (F32.1) bis schwer (F32.2 und F32.3). Die mittelgradige depressive Episode zeichnet sich durch das Vorliegen von mindestens zwei der drei o. g. typischen Symptome einer Depression sowie mindestens drei der anderen häufigen Symptome aus und muss mindestens zwei Wochen andauern, um als solche klassifiziert zu werden (Dilling et al. 2015, 173 f.).
} 
stimmt". Die diffuse Vermutung, welche durch beispielhafte Handlungen von Maria - wie Aufhetzen und Abschotten - unterfüttert wird, erfährt ihre Auflösung in der Feststellung, dass diese "eine immer schlimmer werdende Alkoholikerin" gewesen sei. Tochter und Vater erscheinen dabei beide als Erleidende der Folgen des Alkoholismus von dessen Lebenspartnerin, den sie „leider zu spät“ bemerken. Die Wahl der semantischen Rollen und Prädikate kennzeichnet Paula und ihren Vater als ebenbürtige Verbündete bzw. Erleidende, die dem destruktiven Verhalten von Maria gleichermaßen hilflos ausgeliefert sind. Die Gültigkeit der Charakterisierung von Maria als gefährlich wird auf der interaktiven Ebene untermauert durch das Anführen einer vorangehenden Warnung des Vaters durch dessen Eltern und „viele[r] andere[r] " vor ihr. Im weiteren Verlauf der Erzählung schildert Paula, dass sie durch Marias Intervention drei Jahre von ihrer Mutter getrennt war, welche mittlerweile ein Kind mit ihrem neuen Lebenspartner bekommen hat. Maria habe ihr eingeredet, dass die Mutter sie durch das neue Kind ersetzt, was Paula geglaubt habe. Plastisch schildert sie ihr Erleben, gemeinsam mit ihrem Vater im "verfallenen Haus“ von Maria gefangen zu sein. Es folgt eine Trennung des Vaters von Maria, doch lebten Paula und ihr Vater noch weiterhin in deren Haus. Dabei beschreibt sie die prekäre sozio-ökonomische Situation, in der sich die Beiden befinden. Sie schildert Drohungen von Maria und berichtet von sexualisiert-übergriffigem Verhalten ihres Sohnes. Zwischenzeitlich habe Paula wieder Kontakt zu ihrer Mutter und nach und nach eine Beziehung zu dieser aufgebaut. Im Frühjahr 2016 zogen Paula und ihr Vater schließlich aus dem Haus von Maria aus und in eine neue Wohnung. Auch diesen Übergang beschreibt Paula als entbehrungsreich. Dann fügt sie an:

„Dass Papa weiterhin nach einer Frau suchte, zwingend, und ich mit den Umständen nicht mehr klar kam, verschlechterte sich mein Zustand immer und immer mehr. Albträume und Ängste plagen mich bis heute."

Paula führt hier zwei große Belastungsfaktoren mit Blick auf ihre psychische Gesundheit an: Die Partnersuche des Vaters sowie „die Umstände“, womit hier offensichtlich die prekären und unsicheren Lebensbedingungen, von denen vorangehend berichtet wurde, adressiert sind. Die Partnersuche des Vaters, dessen Handeln als aktiv, aber zugleich getrieben charakterisiert wird, erscheint für Paula bedrohlich. Offensichtlich bewertet sie das Suchen und Finden einer Partnerin durch den Vater - vor dem Hintergrund der vergangenen Erfahrungen - als Gefahr für sich und das familiäre Milieu. Auffallend ist die individualisierende Deutung der eigenen Erkrankung, die Paula als einen eigenen Mangel an Bewältigungskompetenz beschreibt. Der zunehmenden Verschlechterung ihres "Zustandes“ wirkt sie passiv ausgeliefert und erscheint in der Folge als Betroffene von Angstzuständen und Albträumen. In der Schilderung der zugrunde liegenden Problematik (Themenfeld 2a. im Eigenbericht) benennt sie diese als „Depression/Depressionsphasen". Wie stark die Agency- und Identitätsbezüge mit hegemonialen Normalitätsordnungen verknüpft sind, zeigt sich in der biographischen Erzählung von Paula eindrücklich. So werden die Trennung der Eltern, die Auswirkungen der Suchterkrankung der Partnerin des Vaters, das Aufwachsen unter prekären Bedingungen und die eigene psychische Erkrankung nicht nur aufgrund ihrer lebensweltlichen Konsequenzen als belastend und leidvoll erfahren. Darüber hinaus wird die (familiäre) Lebenspraxis auch in Anbetracht inrer Abweichung von gesellschaftlich als Normalität betrachteten Lebensvollzügen und Kontexten mit Narrativen von individuellem Versagen und Scheitern verknüpft (dazu auch: Rein 2020).

\subsubsection{Fallstruktur}

Narrative Identität: In der narrativen Selbstdarstellung zeigt sich eine hohe Divergenz zwischen vergangenem Ich (sorgloses Kind) und gegenwärtigem Ich (kranke, überforderte Jugendliche). Die familiäre Lebenswelt Paulas wird - seit der Trennung der Eltern - von Instabilität, heftigen Konflikten, Manipulation und missbräuchlichem Verhalten dominiert; eng verbunden mit neuen Lebenspartnerschaften der Eltern. Paula beschreibt eine enge, aber zugleich unsichere Bindung zum Vater, der in weiten Teilen der Erzählung als ebenso abhängig und den Bedingungen passiv-ausgeliefert erscheint wie sie 
selbst. Die vergangenen Beziehungserfahrungen führen bei ihr zu einer ängstlich-repulsiven Haltung, die sich in der Erwartung weiterer Katastrophen zeigt. Ihre psychische Erkrankung Depression deutet Paula in der Selbstbeschreibung als mangelnde Resilienz gegenüber den andauernd belastenden Lebensbedingungen.

Agency: Auf der Ebene der Interaktion mit der Leserschaft taucht Paula als kompetente Erzählerin mit hoher epistemischer Autorität auf. Zugleich zeigt sich darin eine Diskrepanz zu ihrer Agentivität in den Erzählsätzen in weiten Teilen ihrer Geschichte. Zwar finden sich dort ebenso Selbstpositionierungen als bewusste und trotz widriger Umstände handlungsfähige Akteurin, doch dominieren passiv-ausgelieferte Agentivierungen die autobiographische Erzählung. In der Geschichtenmoral tritt sie als Opfer belastender Lebensumstände und übergriffiger Handlungsweisen anderer auf, deren Folgen sie schlussendlich nicht mehr standhalten kann, sodass sie mit der Ausbildung psychischer Symptome reagiert.

Lebenspraxis: Im handlungspraktischen Wirklichkeitsvollzug durch Paula zeigen sich immer wieder Handlungsinitiativen und erfolgreiche Krisenbewältigungsprozesse. Doch ist die Lebenspraxis seit der Trennung der Eltern einer so hohen Dichte an traumatischen Krisen ausgesetzt, die nicht durch elterliche Fürsorge aufgefangen werden, dass Paula Ängste ausbildet und mit Überforderung reagiert. ${ }^{4}$ Ihre eigenen Handlungs- und Entscheidungsspielräume sind einerseits durch die brüchigen Umstände in Verbindung mit der prekären sozio-ökonomischen Situation der Familie restringiert; andererseits ist Paula in parentifizierende Dynamiken eingebunden, welche sie ebenso in ihrer Krisenbewältigung überfordern. Mit der Adoleszenzkrise verknüpfte Autonomisierungsbewegungen (Oevermann 2016, 171 f.) werden in Paulas Falle zudem dadurch erschwert, dass eine unsichere Bindung zu den zentralen erwachsenen Bezugspersonen einen stimmigen Ablösungsprozess erschwert, weil diese selbst als stabilisierungsbedürftig erscheinen.

\subsubsection{Fallstrukturhypothese}

Das Zerbrechen der Kernfamilie markiert für die Lebenspraxis von Paula einen biographischen Bruch, dem eine hohe Dichte an traumatischen Krisen in Form von Gewalt, elterlicher Abhängigkeit und materieller Not folgen. Da bereits im Kindesalter keine ausreichende Abmilderung dieser Krisen durch erwachsene Bezugspersonen erfolgt, kommt es - trotz starker Anstrengungen von Paula - zu nicht mehr bewältigbaren Belastungen, die in einer Verletzung der psychischen Integrität der Heranwachsenden resultieren. Dieser Entwicklung entspricht die biographische Kernnarration vom plötzlichen Zerfall einer "heilen“ Lebenswelt, welcher eine negative Verlaufskurve einleitet. Der damit einhergehende Leidensdruck erfährt eine Verdopplung, weil die Krisen(bewältigung) des familialen Milieus ebenso wie die eigene psychische Erkrankung im Abgleich mit hegemonialen Normalitätsvorstellungen - zugleich als ein Scheitern der Lebenspraxis an zentralen gesellschaftlichen Anforderungen erfahren werden.

\subsection{Der Fall Elena}

Elena ist 20 Jahre alt, als sie den Eigenbericht verfasst. Sie hat keine Geschwister, verfügt über einen mittleren Bildungsabschluss und arbeitet aktuell in einem Nebenjob. Sie lebt mit ihrer Mutter und deren Lebenspartner in einer gemeinsamen Wohnung im ländlichen Raum. In der Vergangenheit hat sie einmal eine ambulante Psychotherapie in Anspruch genommen. Zum Erhebungszeitpunkt wird sie ebenfalls mit der Diagnose einer mittelgradigen depressiven Episode (F32.1) in der Jugendpsychosomatik behandelt. Die Erzählung ihrer Lebensgeschichte beginnt sie mit folgender Ausführung:

"Ich bin bis zum 5. Lebensjahr mit beiden Elternteilen aufgewachsen, dann haben sie sich getrennt. Ich war immer lieber alleine als mit

\footnotetext{
${ }^{4}$ Mit der traumatischen Krise bezeichnet Oevermann (2016) jene Krisen, „in der ein konkretes Leben von unvorhergesehenen, überraschenden Ereignissen der äußeren oder inneren Realität unvorbereitet ereilt wird“, auf die es „nicht nicht reagieren kann“ (ebd., 165). Bedeuten diese Krisen eine Überforderung, können sie zu Depression und Rückzug bzw. zu Resignation und Ängstlichkeit führen (ebd., 170).
} 
anderen Kindern und habe viel gemalt und gelesen. Meine Mutter war viel unterwegs und hat viel gearbeitet."

Das gemeinsame Aufwachsen mit beiden Elternteilen und die anschließende Auflösung der elterlichen Beziehung im fünften Lebensjahr Elenas werden als markante Einleitung der biographischen Erzählung gewählt. Auffallend ist die anschließende Rahmung von Alleinsein und Selbstbeschäftigung als Präferenz, womit sich die Erzählerin sogleich agentiv als Einzelgängerin präsentiert. Mit der Beschreibung der häufigen Abwesenheit der Mutter, auch im Zusammenhang mit den ökonomischen Zwängen von Erwerbsarbeit, liefert Elena sodann einen Einblick in das sozialisatorische Milieu, in dem sie fortan aufwächst und welches damit zunächst als von prekären und herausforderungsvollen Bedingungen gezeichnet erscheint. Die Beschreibung selbst wird als Aufzählung von Fakten vorgestellt.

In der Erzählung findet sich dann ein Sprung zum zwölften bis dreizehnten Lebensjahr, wo Elena das Einsetzen einer "rebellischen Phase“ mit Blick auf ihre Schulbiographie beschreibt, einhergehend mit ständigem „Herausfordern“ der Lehrer und einem „drastischen“ Leistungsabfall. Dann merkt sie an:

„Ich wollte zu meinem Vater ziehen, da meine Mutter gewalttätig war und ich mich von ihr gehasst fühlte. Irgendwie kam es zu einem Gerichtsverfahren, wodurch ich fast in ein Kinderheim gekommen wäre. Letztendlich durfte mein Vater keinen Kontakt mehr zu mir haben, weshalb er zurück in [Herkunftsland X] zog."

Die Erzählerin äußert den konkreten Wunsch, zu ihrem Vater zu ziehen. Als Begründung wird in erster Linie das Loskommen von der Mutter angeführt, welche Elena als "gewalttätig“ charakterisiert, womit sie dieser sogleich eine Täterinnenrolle zuweist. Sowohl Elena als auch die Mutter treten in der Erzählung zunächst agentiv auf und die Agentivierungen werden auf der Interaktionsebene mit Anspruch auf Faktizität vermittelt. Dann findet sich erstmals eine Subjektivierung ihrer Deutung, als Elena anmerkt, dass sie sich von ihrer Mutter gehasst fühlte. Sie stellt sich als Erfahrende in den Vordergrund, wobei das starke Prädikat auf eine hohe Intensität im Erleben von Elena verweist. Der anschlieBende Erzählsatz sticht durch seine Diffusität hervor, da weder konkrete Akteur*innen benannt, noch die weiteren Hintergründe des angeführten Gerichtsverfahrens erläutert werden und auch die epistemische Markierung auf Unsicherheit in der konkreten Realitätskonstruktion durch die Erzählerin verweist. Dies lässt sich zugleich als sprachlicher Ausdruck eines erlebten Agency-Verlusts durch die plötzlich drohende Herausnahme des Kindes aus dem familiären Umfeld, aus für es nicht ganz durchschaubaren Gründen, lesen. Dafür spricht auch, dass Elena nun in der semantischen Rolle der Betroffenen erscheint. Als Ergebnis des Gerichtsverfahrens präsentiert sie ein Umgangsverbot des Vaters mit ihr. Dessen anschließende (Re-)Migration in sein Herkunftsland führt sie als direkte kausale Folge dieses Beschlusses an - mit hohem Anspruch auf Faktizität.

„Danach entwickelte ich allmählich eine soziale Phobie und eine Depression, ich hatte viele Selbstmordgedanken, und habe mich zurückgezogen, bis ich gar nicht mehr/sehr selten zur Schule ging, weshalb ich nach der achten Klasse entweder hätte sitzen bleiben müssen oder auf eine Realschule in die neunte hätte versetzt werden können. Ich entschied mich für das zweite und es wurde nicht besser."

Elena beschreibt, wie sie im Anschluss an diese Vorgänge „allmählich“ psychische Erkrankungen entwickelte. Soziale Phobie und Depression erscheinen durch die temporale Verknüpfung in ihrer Erzählung als psychische Bewältigungsreaktion der anhaltend belastenden Lebensumstände - mit dem Verlust des Vaters als einschneidendem Ereignis in ihrer Biographie. Sie beschreibt Symptome der Erkrankungen sowie Vermeidungsverhalten und dessen schulische Konsequenzen. Dabei weisen die semantischen Rollen und Aktionsprädikate sie eher als mitbeteiligte Person denn als ausschließlich Erleidende aus. Lediglich den sich verengenden schulischen Handlungsoptionen sieht sie sich dann passiv ausgesetzt. Dennoch beschreibt sie auch hier Handlungsalternativen ebenso wie sie das aktive Fällen einer Entscheidung zugunsten eines Schulwechsels hervorhebt. Doch weist sie sogleich darauf hin, dass 
sich diese Entscheidung nicht als verbessernd bewährt. Auffallend in der biographischen Erzählung ist, dass die Formulierung „und es wurde nicht besser" in ähnlicher Form bereits zuvor auftaucht. Auch dort geht es um eine Entscheidungssituation bezüglich eines Schulwechsels. Unklar bleibt in beiden Fällen, was genau sich nicht verbessert. Denkbar wären Schulleistungen, das Verhältnis zu Mitschüler*innen, ihr psychisches Wohlbefinden oder ganz allgemein ihre Lebenssituation, für die sie sich durch einen Wechsel Verbesserungen erhofft, die jedoch nicht eintreten. Insgesamt scheint sich diese wiederholte Erfahrung in einem Pessimismus mit Blick auf die Wirkmächtigkeit eigener Handlungen und Entscheidungen - zur Verbesserung des eigenen Lebens - zu verfestigen, der sich hier sprachlich manifestiert.

"Ich ging nach dem Realschulabschluss wieder auf das Gymnasium, wo es mir nach anfänglichem Enthusiasmus wieder sehr schlecht ging und oft nicht hin ging, weshalb ich die Klasse wiederholen musste. Das habe ich dann auch, ich wurde versetzt und brach nach dem ersten Halbjahr die Schule ab, mit dem eigentlichen Ziel, einerseits von der empfundenen Qual wegzukommen, andererseits eine Selbstmordplanung und -umsetzung zu erstreben."

Ohne weitere Ausführungen zur Situation während des Realschulbesuchs merkt Elena in einem Beisatz an, die Mittlere Reife absolviert zu haben, der ein erneuter Besuch des Gymnasiums folgt. Ihre agentiven Leistungen werden dabei in den Hintergrund gestellt; der Realschulabschluss erscheint als einfacher Vorgang. Auch in dieser Erzählung findet sich die Dynamik eines begeisterten, hoffnungsvollen Starts in der neuen Schule, der jedoch schnell in einer erneuten psychischen Krise, verbunden mit Schulabsentismus, dem Wiederholen einer Klassenstufe und schließlich dem Schulabbruch, mündet. Elena erscheint in den Agentivierungen - nun deutlicher als zuvor - als Erleidende der Folgen der psychischen Erkrankung. Agentiv präsentiert sie sich allerdings mit Blick auf den Schulabbruch, den sie als intentionalen Plan mit einem konkreten Ziel beschreibt: Sie habe die Schule beendet, um von den empfundenen Qualen eine nochmalige Subjektivierung des geschilderten Erlebens - fortzukommen. Zugleich habe sie „eine Selbstmordplanung und -umsetzung erstreben" wollen. Das Vorhaben wird hier als konkreter Gestaltungsakt mit dem Ziel eines erfolgreich vollzogenen Suizids präsentiert und erscheint damit zunächst als agentive Bewältigungsleistung der Erzählerin, die im Umgang mit dem eigenen Leiden und letztlich ihrem eigenen Leben eine "finale" Entscheidungs- und Handlungsmächtigkeit zum Ausdruck bringt. Zugleich wird in der Erzählung deutlich, dass dieses Vorhaben das Resultat eines als übermächtig erlebten Leidensdrucks und wiederholten AgencyVerlusts ist, denen sie mit einem endgültigen Ausstieg aus dem sozialen Leben ein Ende zu setzen trachtet.

„Momentan arbeite ich immer noch als Kurierin und fange vermutlich bald eine Ausbildung in einem Berufsförderwerk an."

Mit Blick auf die vorangehende Ausführung ist der plötzliche Wechsel von der Suizidplanung von der hier keine explizite Distanzierung erfolgt - zum Thema des aktuellen Beschäftigungsverhältnisses und dem möglichen Beginn einer Ausbildung besonders auffallend. Die Perspektivänderung von der Selbstauslöschungsabsicht hin zur Schilderung von zukünftigen Bildungsvorhaben erfährt keine weitere Erläuterung. Eine schlüssige Erklärung hierfür würde die zwischenzeitliche Hinwendung zum psychosozialen Hilfesystem sowie die damit verbundene Übergangssituation bilden. So erscheint es plausibel, dass sich Elena vom dem Klinikaufenthalt Hilfen zu alternativen Krisenbewältigungsstrategien und eine (Wieder-)Herstellung von Agency erhofft, um doch noch prospektive Lebens- und Bildungsentwürfe verwirklichen zu können. Besonders deutlich zeigt sich hier ein Spannungsverhältnis zwischen iterativen und projektiven Bezügen auf Agency (Emirbayer/Mische 2017).

\subsubsection{Fallstruktur}

Narrative Identität: In der Handlungs- und Selbstdarstellung taucht Elena fast ausschließlich als Einzelgängerin und Einzelkämpferin gegen eine Welt auf, die ihr feindselig-repulsiv gegenübersteht. In der Erzählung ihrer Lebensgeschichte dominieren Narrative von Scheitern und Leiden. Weder in der familiären noch in der 
außerfamiliären Lebenswelt von Schule und Peer-Group findet sie soziale Ressourcen, Anerkennung und Zugehörigkeit. Stattdessen erlebt sie sowohl von ihren Eltern als auch von Mitschüler*innen Ablehnung, Gewalt, Trennung und Verlust. Elena beschreibt sich als rebellierende, unbeliebte Schülerin mit häufigen Schulwechseln, die - trotz eines erfolgreichen mittleren Bildungsabschlusses - nicht über die notwendige „Wehrhaftigkeit“ (Wernet 2018, 247 ff.) verfügt, um sich dauerhaft in den Sphären von Leistung und Gleichaltrigengruppe behaupten zu können. Depression erscheint in ihrer Erzählung als sich allmählich entwickelnde Überforderungsreaktion auf die beschwerlichen und kaum mehr bewältigbaren Lebensumstände.

Agency: Mit Blick auf die semantischen Rollen in den Erzählsätzen fällt auf, dass sich Elena von Beginn an - auch in den Erzählungen über das Kindesalter - als selbständig handelnde Akteurin markiert. Im Erzählverlauf bildet sich dann allerdings ein Muster ab, dass die geringe Wirkmächtigkeit eigener Handlungen und Entscheidungen in den Vordergrund stellt, indem Handlungs- und Veränderungsinitiativen immer wieder als wirkungslos, nicht zielführend oder nicht realisierbar resümiert werden. Äußere Umstände werden durchgehend als diffus, unkontrollierbar und ablehnend-destruktiv charakterisiert; allen anderen Akteur*innen in der Erzählung werden feindselige oder im besten Falle nicht-unterstützende Attribute zugeordnet. Auf der Interaktionsebene zeigt sich Elena mit Blick auf Agentivierungskonstruktionen, Bewertungskompetenz und Deutungsmacht als überwiegend sichere Erzählerin mit hoher epistemischer Autorität. In der Geschichtenmoral tritt sie als Erleidende extrem belastender sozialer Umstände in ihrer unmittelbaren Lebenswelt auf, welche sich trotz aller Anstrengungen ihrerseits nicht zu ändern scheinen, was immer wieder zu Rückzug, Depression, Ängsten, bis hin zu Suizidabsichten bei ihr führt.

Lebenspraxis: Die Lebenspraxis von Elena zeichnet sich - ebenso wie die von Paula - durch eine dichte Aneinanderreihung von traumatischen Krisen aus, die vom sozialisatorischen Milieu nicht oder nur unzureichend abgefedert werden. Die Strukturlogik des „Sprechaktes des Versprechens", dass es im Zweifelsfall schon gut gehen wird (Oevermann 2016, 166), kann von diesem Milieu nicht adäquat vermittelt werden, da es selbst von permanenten Brüchen, Unsicherheiten und Gewalt gekennzeichnet ist. Auch im auBerfamiliären Umfeld der Peer-Group findet Elena keine korrigierenden Erfahrungen zur familiären Lebenswelt. Die Lösung von Entscheidungskrisen findet immer wieder durch Rückzug und Abbruch statt und dort, wo Entscheidungen getroffen werden, scheinen sich die Lösungen im Nachgang nicht als stimmig zu bewähren. Dies führt letztlich zu einer Haltung die sich mit Oevermann als struktureller Pessimismus (ebd., 164) kennzeichnen lässt (,es wird einfach nicht besser"), die in weiterem Rückzug und Resignation mündet und dazu führt, dass in der primären Lebenspraxis von Elena dann fast ausschließlich auf selbst-schädigende Krisenbewältigungsstrategien zurückgegriffen wird und letztlich Instanzen der stellvertretenden Krisenbewältigung angerufen werden.

\subsubsection{Fallstrukturhypothese}

Die Selektion der Lebenspraxis verweist auf die Bewältigung von Entscheidungs- und traumatischen Krisen durch den Vollzug starker Brüche, die sich im Rückblick der Erzählerin wiederholt nicht bewähren und/oder sich als (weiter) schädigend erweisen, wodurch die Wirkmächtigkeit transformatorischer Handlungen zur Verbesserung des eigenen Lebens zunehmend gering bewertet wird. Die biographische Kernnarration beschreibt einen fortschreitenden Resignationsprozess der Einzelkämpferin Elena gegenüber einer ihr feindlich begegnenden Welt, der jedoch bis zur Abschlusssequenz durch projektive Zukunftsentwürfe irritiert wird. In diesen scheint Agency als Veränderungs- und Gestaltungspotential durch, das erst in der Distanz zur etablierten Routine von Rückzug und radikalem (Beziehungs-)Abbruch ermöglicht wird.

\section{FAZIT}

Der Beitrag unternimmt den Versuch einer Synthese pragmatistisch-strukturalistischer wie konstruktivistischer Zugänge zur Agency Adoleszenter im Kontext der psychischen Erkrankung Depression. Die Brücke bildet eine relationale Perspektive, die dem Akteur*innebe- 
griff weiterhin eine zentrale Gewichtung beimisst, wenngleich sie diesen und dessen Agency vor allem als in sozialen Beziehungen hervorgebrachte begreift. Mit dieser Perspektive erfolgt eine kritische Bezugnahme auf aktuelle AgencyDiskurse zum Thema psychische Erkrankung. Die vorliegenden empirischen Arbeiten legen einen starken Fokus auf subjektive Sinnzusammenhänge und deren diskursive Hervorbringung (in Netzwerkgeflechten) (z. B. Hoffmann 2012, 2015; Deppermann 2015). Mit Blick auf AgencyErleben und -Zuschreibung sowie die interaktive Herstellung von Identität ergeben sich dadurch wichtige Erkenntnisse für das Verständnis psychischer Erkrankungen aus Sicht der Adressat*innen psychosozialer Hilfen. So gelingt es auch in der vorliegenden Analyse, Depression als vom psychosozialen Hilfesystem bereitgestelltes Narrativ zu rekonstruieren, welches den Adoleszenten in situationalen Erfahrungen innerhalb ihres sozialen Netzwerks vermittelt wird und dann als Teilidentität Anschluss findet (Keupp et al. 2008, 218). Diese erfüllt eine doppelte Funktion: Nach innen hin, indem sie jene spezifischen familiären, schulischen, Peer- und Selbst-Erfahrungen wie Leidenszustände unter einer anerkannten Kategorie gesundheitlicher Beeinträchtigung für die beiden Akteur*innen kohärenzstiftend integriert; nach außen hin, indem sie zu deren sozialer Verortung, z. B. im Bedeutungsnetz der Institutionen des (psychosozialen) Hilfesystems, beiträgt (ebd., 243 ff.). Zugleich verweist die Fallanalyse allerdings darauf, dass es verkürzt wäre, Depression ausschließlich als Narrativ bzw. narrative Teilidentität zu begreifen. Denn darüber hinaus zeigt sich in beiden Fallvignetten, dass die mit dem Begriff verbundenen Phänomene eng mit psychophysischen wie auch sozialen Reaktionen auf anhaltende Belastungen und Überforderungen in für die beiden Akteurinnen bedeutsamen Beziehungsgefügen verknüpft sind, woraus sich ebenso Konsequenzen für deren Handlungsfähigkeit und Wirkmächtigkeit ableiten. So lässt sich Agency durch die Analyse der Krisenbewältigungsprozesse (Oevermann 2000, 2016) zudem in aus diesen hervorgehenden Handlungsroutinen rekonstruieren, welche - vor dem Hintergrund relational hergestellter Selbstrepräsentationen sowie der aktuellen sozialen Einbettung der Erzähler*innen - rigide aufrechterhalten werden und sich im So- zialisationsprozess dann als dysfunktional erweisen, weil sie die Etablierung von neuen, die Autonomie der Lebenspraxis fördernden Krisenbewältigungsstrategien beschränken. Umgekehrt wirkt dies wiederum auf Identitätsbildungsprozesse zurück. Agency-Herstellung und -Transformation finden also sowohl auf der Ebene von Identitätsbildung und -sicherung wie auch im Wirklichkeitsvollzug der Lebenspraxis statt und sind überhaupt nur in der wechselseitigen Verschränkung dieser und mit diesen differenziert zu erfassen. Zugleich wird in der vergleichenden Einzelfallanalyse deutlich, dass sich diese reziproken Interaktionsprozesse innerhalb eines sozialen Raums vollziehen, der durch Machtverhältnisse gekennzeichnet ist (Bourdieu 1985; Keupp et al. 2008, 198 ff.). So finden sich in beiden autobiographischen Erzählungen individualisierende Deutungen der eigenen Erkrankung als ein Mangel an Bewältigungsfähigkeit. Depression erscheint in den Eigenberichten damit als ein individuelles Scheitern an den "normalen“ Ansprüchen moderner Gesellschaften nach Selbstbestimmung und -verwirklichung, Erfolg, Glück und Teilhabe (Ehrenberg 2015). Die umrissenen sozio-ökonomischen Verhältnisse, unter denen die Adoleszenten aufwachsen und ihre Erkrankungen ausbilden, zeichnen jedoch ein anderes Bild: So belegen repräsentative Langzeitstudien zur gesundheitlichen Lage von Kindern und Jugendlichen (Lampert et al. 2019) die massiv ungleichen Chancen auf Gesundheit zuungunsten sozioökonomisch benachteiligter Heranwachsender. Dies zeigt sich am stärksten im Bereich der psychischen Gesundheit (vgl. ebd.). Das Narrativ des (selbstverschuldeten) Versagens lässt sich vor diesem Hintergrund als ein Hinweis auf die symbolisch-machtvollen Ordnungen innerhalb eines Leistungs- und Gesundheitssystems verstehen, das (zunächst) die Jugendlichen, jungen Erwachsenen und deren Familien in ihrer Eigenverantwortung anruft. Bereits Rainer Döbert et al. $(1980,15)$ weisen darauf hin, dass die Ausbildung von Identitätsformationen und übergreifenden Lebensorientierungen immer auch mit einem Anknüpfen an herrschende kulturelle Überlieferungen zusammenhängt. Wie sich solche Überlieferungen relational vermitteln, das lässt sich zumindest teilweise mit Zugängen wie dem hier vorgestellten analysieren, indem rekonstruiert wird, auf welche Weise depressive Identitäten - 
an der Schnittstelle von primärer Lebenspraxis und stellvertretender Krisenbewältigungsinstanz - narrativ (re-)produziert und (re-)konfiguriert werden. Zugleich können die analytischen Grenzen des präsentierten Ansatzes als Hinweis auf weiterführende Perspektiven, etwa durch die Verknüpfung von fallrekonstruktiven und netzwerkanalytischen Verfahren, gelesen werden. Für zukünftige Forschungsarbeiten zum Thema wäre es zudem spannend, einen Blick darauf zu werfen, wie in den konkreten Interaktionen im Kontext psychosozialer Hilfen solchen Narrativen begegnet wird, die zu einer Privatisierung von Stress und Überforderung (Fisher 2013) beitragen.

\section{LITERATUR}

Bauer, Ulrich (2016): Keine Gesinnungsfrage. Der Subjektbegriff in der Sozialisationsforschung. In: Geulen, DieterNeith, Hermann (Hrsg.): Sozialisationstheorie interdisziplinär. Aktuelle Perspektiven. Reprint. Oldenburg: De Gruyter, 61-91.

Bourdieu, Pierre (1985): Sozialer Raum und „Klassen“. Leçon sur la leçon. Zwei Vorlesungen. Frankfurt am Main: Suhrkamp.

Döbert, Rainer/Habermas, Jürgen/NunnerWinkler, Gertrud (1980): Zur Einführung. In: Dies. (Hrsg.): Entwicklung des Ichs. 2. Auflage. Königstein im Taunus: Verlagsgruppe Athenäum, Hain, Scriptor, Hanstein, 9-30.

Deppermann, Arnulf (2015): Agency in Erzählungen über Gewalterfahrungen in Kindheit und Jugend. In: Scheidt, Carl Eduard/Lucius-Hoene, Gabriele/Stukenbrock, Anja/Waller, Elisabeth (Hrsg.): Narrative Bewältigung von Trauma und Verlust. Heidelberg: Schattauer, 64-75.

Ehrenberg, Alain (2015): Das erschöpfte Selbst: Depression und Gesellschaft in der Gegenwart. Frankfurt am Main: Campus-Verlag.

Emirbayer, Mustafa/Goodwin, Jeff (2017): Netzwerkanalyse, Kultur und das Agency-Problem. In: Löwenstein, Heiko/Emirbayer, Mustafa (Hrsg.): Netzwerke, Kultur und Agency. Problemlösungen in relationaler Methodologie und Sozialtheorie. Weinheim: Beltz Juventa, 286335.
Emirbayer, Mustafa/Mische, Ann (2017): Was ist Agency? In: Löwenstein, Heiko/Emirbayer, Mustafa (Hrsg.): Netzwerke, Kultur und Agency. Problemlösungen in relationaler Methodologie und Sozialtheorie. Weinheim: Beltz Juventa, 138-209.

Esser, Florian/Baader, Meike S./Betz, Tanja/Hungerland, Beatrice (2016): Reconceptualising Agency and Childhood. New Perspectives in Childhood Studies. New York: Routledge.

Fisher, Mark (2013): Kapitalistischer Realismus ohne Alternative? Eine Flugschrift. Hamburg: VSA-Verlag.

Garz, Detlef/Raven, Uwe (2015): Theorie der Lebenspraxis. Einführung in das Werk Ulrich Oevermanns. Wiesbaden: Springer VS.

Grundmann, Matthias (2017): Agency und Sozialisation. In: Löwenstein, Heiko/Emirbayer, Mustafa (Hrsg.): Netzwerke, Kultur und Agency. Problemlösungen in relationaler Methodologie und Sozialtheorie. Weinheim/Basel: Beltz Juventa, 251-269.

Hapke, Ulfert/Cohrdes, Caroline/Nübel, Julia (2019): Depressive Symptomatik im europäischen Vergleich - Ergebnisse des European Health Interview Survey (EHIS). In: Journal of Health Monitoring, 4 (4), https://edoc.rki.de/handle/176904/6242 (31.07.2020).

Hoffmann, Heiko (2011): Das Handeln der Behandelten. Eine rekonstruktionslogische Analyse der Agency von Psychoseerfahrenen. Freiburg: FEL Verlag.

Hoffmann, Heiko (2012): Macht - Wahn - Sinn. Die sequenzielle Analyse von Agency am Beispiel der Darstellung Psychoseerfahrener. In: Bethmann, Stephanie/Helfferich, Cornelia/Hoffmann, Heiko/Niermann, Debora (Hrsg.): Agency. Qualitative Rekonstruktionen und gesellschaftstheoretische Bezüge von Handlungsmächtigkeit, Weinheim/Basel: Beltz Juventa, 181-209.

Hoffmann, Heiko (2015): Borderline-Interaktionen. Komplexe Verflechtungen der Agency in Netzwerken sozialer Unterstützung. Wiesbaden: Springer VS.

Dilling, Horst/Mombour, Werner/Schmidt, Martin H. (Hrsg.) (2015): ICD-10. Internationale Klassifikation psychischer Störungen. Kapitel $\vee(F)$. Klinisch-diagnostische Leitlinien. Bern: Hogrefe. 
Hungerland, Beatrice/Kelle, Helga (2014): Kinder als Akteure - Agency und Kindheit. Einführung in den Themenschwerpunkt. In: Zeitschrift für Soziologie der Erziehung und Sozialisation, 34 (3), 227-232.

Keupp, Heiner (2000): Identität. In: Spektrum Akademischer Verlag (Hrsg.): Lexikon der Psychologie. Online verfügbar unter: https://www.spektrum.de/lexikon/psychologie/identitaet/6968 (31.07.2020).

Keupp, Heiner (2005): Identität. In: Otto, HansUwe/Thiersch, Hans (Hrsg.): Handbuch Sozialarbeit, Sozialpädagogik. München/Basel: Reinhardt, 804-810.

Keupp, Heiner/Ahbe, Thomas/Gmür, Wolfgang/Höfer, Renate/Mitzscherlich, Beate/Kraus, Wolfgang/Straus Florian (2008): Identitätskonstruktionen. Das Patchwork der Identitäten in der Spätmoderne. Reinbek bei Hamburg: Rowohlt Taschenbuch Verlag.

Lampert, Thomas/Hoebel, Jens/Kuntz, Benjamin/Finger, Jonas D./Hölling, Heike/Lange, Michael/Mauz, Elvira/Mensink, Gert/PoethkoMüller, Christina/Schienkiewitz, Anja/Starker, Anne/Zeiher, Johannes/Kurth, Bärbel-Maria (2019): Gesundheitliche Ungleichheiten bei Kindern und Jugendlichen in Deutschland - Zeitliche Entwicklung und Trends der KiGGS-Studie. In: Journal of Health Monitoring, 4 (1),16-40. DOI $10.25646 / 5867$

Leipold, Bernhard/Greve Werner (2015): Sozialisation, Selbstbild und Identität. In: Hurrelmann, Klaus/Bauer, Ullrich/Grundmann, Matthias/Walper, Sabine (Hrsg.): Handbuch Sozialisationsforschung. 8. Auflage, Weinheim/Basel: Beltz.

Lucius-Hoene, Gabriele (2012): „Und dann haben wir's operiert". Ebenen der Textanalyse narrativer Agency-Konstruktionen. In: Bethmann, Stephanie/Helfferich, Cornelia/Hoffmann, Heiko/Niermann, Debora (Hrsg.): Agency. Qualitative Rekonstruktionen und gesellschaftstheoretische Bezüge von Handlungsmächtigkeit. Weinheim/Basel: Beltz Juventa, 40-70.

Lucius-Hoene, Gabriele/Deppermann, Arnulf (2002): Rekonstruktion narrativer Identität. Ein Arbeitsbuch zur Analyse narrativer Interviews. Wiesbaden: Springer Fachmedien.
Oevermann, Ulrich (2000): Die Methode der Fallrekonstruktion in der Grundlagenforschung sowie der klinischen und pädagogischen Praxis. In Kraimer, Klaus (Hrsg.): Die Fallrekonstruktion. Frankfurt am Main: Suhrkamp, 58-153.

Oevermann, Ulrich (2002): Klinische Soziologie auf der Basis der Methodologie der objektiven Hermeneutik. Manifest der objektiv hermeneutischen Sozialforschung. Online verfügbar unter: http://www.ihsk.de/publikationen/Ulrich_Oevermann-Manifest_der_objektiv_hermeneutischen_Sozialforschung.pdf (31.07.2020).

Oevermann, Ulrich (2009): Biographie, Krisenbewältigung und Bewährung. In: Bartmann, Sylke/Fehlhaber, Axel/Kirsch, Sandra/Lohfeld, Wiebke (Hrsg.): ,Natürlich stört das Leben ständig - Perspektiven auf Entwicklung und Erziehung. Wiesbaden: Springer VS, 35-55.

Oevermann, Ulrich (2016): Sozialisation als Prozess der Krisenbewältigung. In Geulen, DieterNeith, Hermann (Hrsg.): Sozialisationstheorie interdisziplinär. Aktuelle Perspektiven. Reprint. Oldenburg: De Gruyter, 155-181.

Rein, Angela (2020): Normalität und Subjektivierung. Eine biographische Studie im Übergang aus der stationären Jugendhilfe. Bielefeld: transcript.

Riemann, Gerhard (1987): Das Fremdwerden der eigenen Biographie: narrative Interviews mit psychiatrischen Patienten. München: Wilhelm Fink Verlag.

Schleiffer, Roland (2010): Kinder und Jugendliche in Institutionen der psychosozialen Versorgung. In: Krüger, Heinz-Hermann/Grunert, Cathleen (Hrsg.): Handbuch Kindheits- und Jugendforschung. Wiesbaden: Springer VS, 891-910.

Schütze, Fritz (1982): Narrative Repräsentation kollektiver Schicksalsbetroffenheit. In: Lämmert, Eberhard (Hrsg.): Erzählforschung. Ein Symposium. Stuttgart: J. B. Metzler, 568-590.

Steffen, Annika/Akmatov, Manas K./Holstiege, Jakob/Bätzing, Jörg (2019): Diagnoseprävalenz psychischer Störungen bei Kindern und Jugendlichen in Deutschland: eine Analyse bundesweiter vertragsärztlicher Abrechnungsdaten der Jahre 2009 bis 2017. In: Zentralinstitut für die kassenärztliche Versorgung in Deutschland (Zi): Versorgungsatlas-Bericht Nr. 18/07 (Version 2). 
Online verfügbar unter: https://www.versorgungsatlas.de/themen/alle-analysen-nach-datum-sortiert/?tab=6\&uid=93 (31.07.2020).

Steffens, Tomas (2004): Familienmilieu und biographische Verläufe psychisch Kranker. FallanaIysen zur sozialen Sinnstrukturiertheit schizophrener Erkrankungen. Frankfurt am Main: Humanities Online.

Wernet, Andreas (2018): Entgrenzung. In: Proske, Matthias/Rabenstein, Kerstin (Hrsg.): Kompendium Qualitative Unterrichtsforschung.
Unterricht beobachten - beschreiben - rekonstruieren. Bad Heilbrunn: Verlag Julius Klinkhardt, 240-256.

World Health Organisation (WHO) (2019): Adolescent Mental Health. Online verfügbar unter: https://www.who.int/news-room/factsheets/detail/adolescent-mental-health (31.07.2020).

\section{Zum Autor}

Christian Armbrüster ist wissenschaftlicher Mitarbeiter am Institut für Erziehungswissenschaft der Johannes Gutenberg-Universität Mainz. Seine Forschungsinteressen liegen im Bereich der Kindheits- und Jugend- sowie der Qualitativen Sozialforschung. Schwerpunktmäßig befasst er sich mit den Themen Biographie, Sozialisation, Identität und Agency.

\section{Kontakt}

Christian Armbrüster, M.A. Institut für Erziehungswissenschaft Johannes Gutenberg-Universität Mainz Georg Forster-Gebäude Jakob-Welder-Weg 12 55128 Mainz

E-Mail: armbruester@uni-mainz.de URL: https://www.sozialpaedagogik.fb02.uni-mainz.de/team/christian-armbruester-m-a/ 